\title{
A METHOD FOR IMPLEMENTING FORCE-LIMITED VIBRATION CONTROL
}

\author{
Daniel B. Worth \\ NASA Goddard Space Flight Center \\ (301) 286-5140 \\ daniel.worth@gsfc.nasa.gov
}

\begin{abstract}
NASA/GSFC has implemented force-limited vibration control on a controller which can only accept one profile. The method uses a personal computer based digital signal processing board to convert force and/or moment signals into what appears to be an acceleration signal to the controller. This technique allows test centers with older controllers to use the latest force-limited control techniques for random vibration testing. The paper describes the method, hardware, and test procedures used. An example from a test performed at NASA/GSFC is used as a guide.
\end{abstract}

\section{INTRODUCTION}

\section{Overview of Force Limiting}

During a conventional random vibration test, a slip table or a shaker head is driven to a specified acceleration power spectrum. The specification is usually derived from an envelope of flight tests with similar payloads. This conservative approach insures that an undertest will not occur. In some frequency bands, this approach produces a significant overtest - especially at the fixed-base natural frequencies of the test item. When the test item (space craft or instrument) is mounted on the launch vehicle or spacecraft, the item will act as a frequency absorber near the region of fixed base natural frequency. In other words, peak accelerations and possibly damage will occur during testing at certain frequencies which in flight will be sharply reduced and will show up as dips in the spectrum.

Force limiting is an attempt to reduce the effectively infinite impedance of the slip table or shaker head by limiting the interface forces. At the frequencies where the test item will resonate and act like an absorber, the interface force will limit the vibration level and produce a dip in the acceleration spectrum. One could also limit based on moments or any other measured phenomenon. Methods for determining the force limiting spectrum are described in various papers $[1,2,3]$.

\section{System Description}

Figure 1 is a sketch of a typical force-limiting control system. The outer loop, consisting of accelerometers, charge amplifiers, controller, drive amplifier, and shaker, is the conventional capability of any vibration control system. Force-limiting adds a force gage conditioning system, shown in the shaded boxes of figure 1 . The force gages are added at the interface of the test item and the shaker head or table. The signals are conditioned by charge amplifiers and the force gage signal processing system. The signals are then sent to the controller.

The signal from the force gages is compared to a limit spectrum. Some controllers cannot handle more then one spectrum. The system discussed in this paper is designed to allow test facilities with such controllers to test using force limiting. The system described in this paper shapes the force gage signal into an acceleration signal that can be compared to the same specification used for the accelerometers. This system is described in detail in the following sections. 


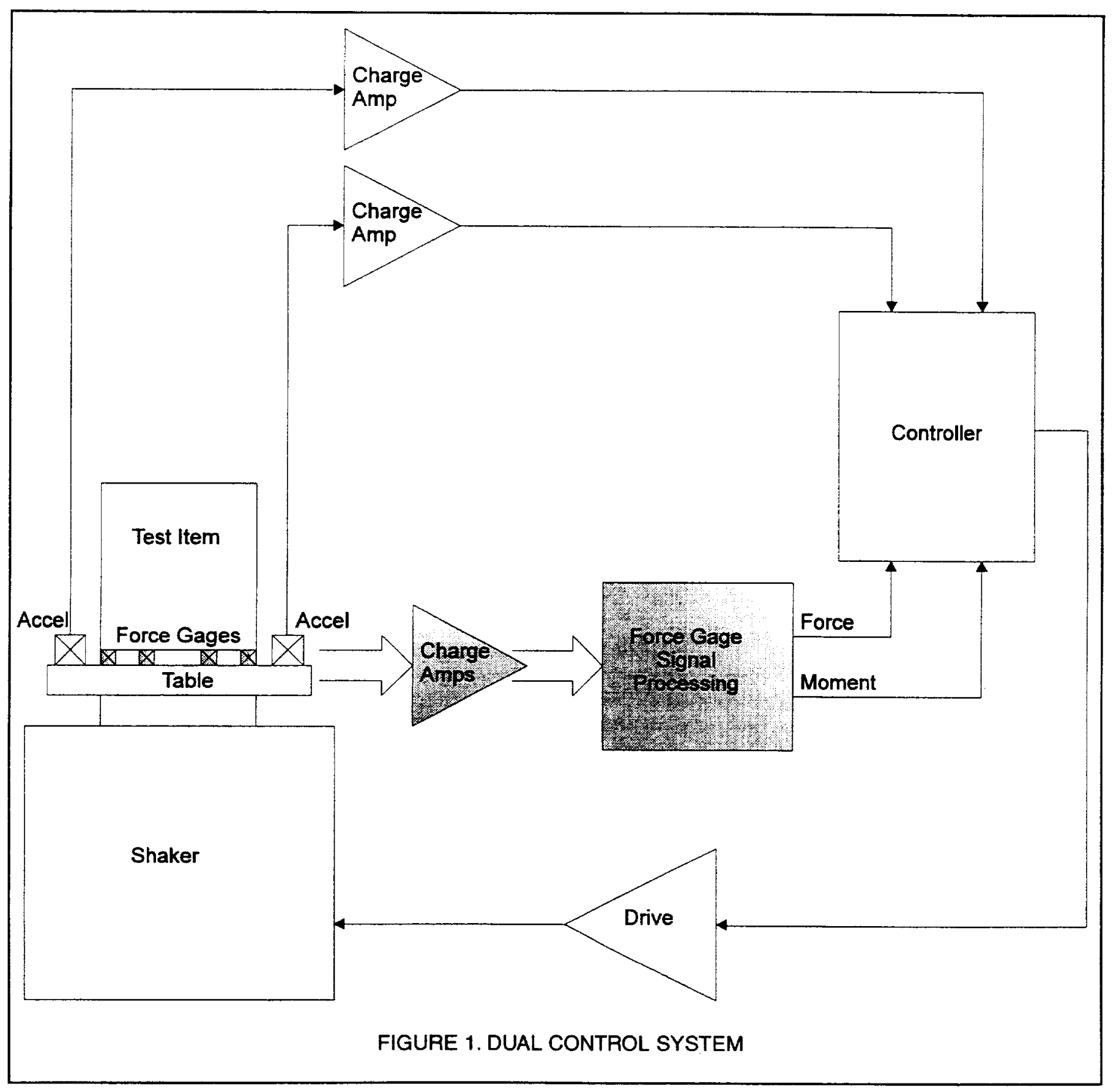

\section{FORCE GAGE SIGNAL PROCESSING SYSTEM}

A drawing of the force gage signal processing system is shown in figure 2. This system is designed to handle up to eight triaxial force gages, but can be expanded to a larger number by the addition of more Sum and Difference amplifiers. The number of force gages and the wiring between the Sum and Difference Amplifiers and the Digital Signal Processing (DSP) boards can be changed depending on the test configuration. Figure 2 is wired for a case where six triaxial force gages are used to measure six degrees of freedom - three translational forces and three moments. 


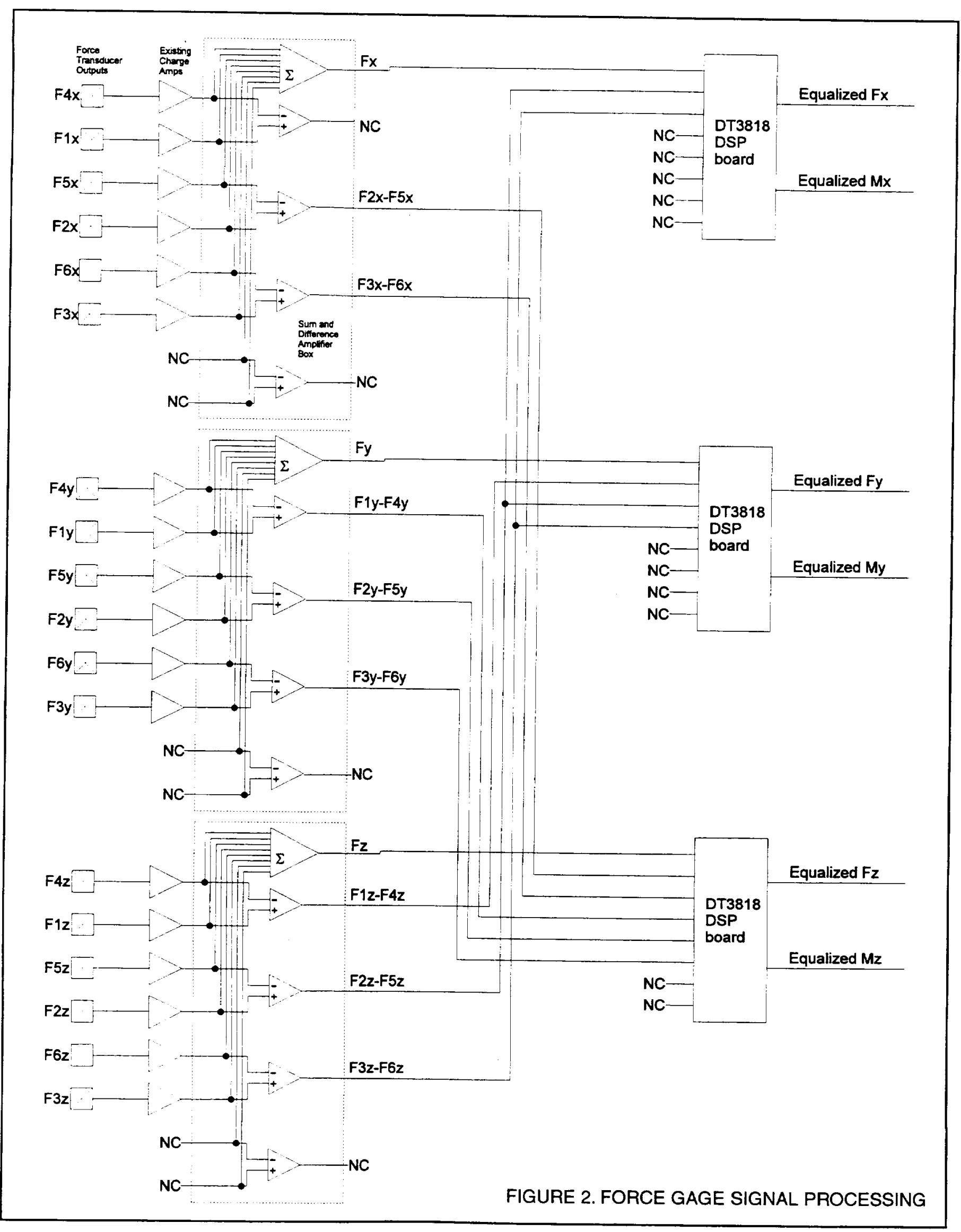


Each force gage output is conditioned using charge amplifiers. Charge attenuators are sometimes used between the force transducers and the charge amplifier to prevent saturation of the amplifier input signal or clipping of the amplifier output signal.

The signals from each charge amplifier are fed into Sum and Difference Amplifier boxes. These boxes, as the name implies, sum all of the signals fed into the box and also compute differences between symmetrical pairs of conditioned force gage outputs. These computed difference signals are used to calculate moments. If no moments are to be calculated, or no individual monitoring of each force gage is desired, then a simple charge summing device can be used instead of the Sum and Difference Amplifier.

\section{TABLE 1. SPECIFICATIONS OF DSP BOARD}

\section{Data Translation DT3818 DSP board (Fulcrum)}

Texas Instruments TMS320C40 DSP @ 50 MHz

Eight Analog Inputs

A 16 bit Analog/Digital (A/D) converter for each channel

1 to 52 kilosamples per second maximum sampling rate per channel

Anti-Aliasing Filters for each channel

Two Analog Outputs

A 16 bit Digital/Analog (D/A) converters for each channel

Reconstruction Filter for each output channel

Data Translation DSP-EZ software runs on the board

Basic language programming of TMS320C40

Mathematical manipulation of buffers

Highpass, Lowpass, and Bandpass Filtering available

User defined filter

Fast Fourier Transform (FFT) and Inverse FFT (IFFT)

Visual Basic VBX control runs on the Personal Computer

Properties of VBX control operate the A/D converter, D/A converter, and execute the DSP-EZ program.

The outputs of each Sum and Difference Amplifier Box are then routed to the DSP boards for scaling and equalization. The specifications of the DSP board are shown in table 1. The DSP board front-end amplifier and mixer provides scaling of each symmetrical pair of force gage signals to compute a given moment, or sum multiple gage outputs to produce a force sum. The front-end of the processing is shown in figure 3 . The board accepts up to eight inputs (label F1 through F8 in figure 3) and computes two outputs (labeled $\mathbf{F}$ and $\mathrm{M}$ ). 


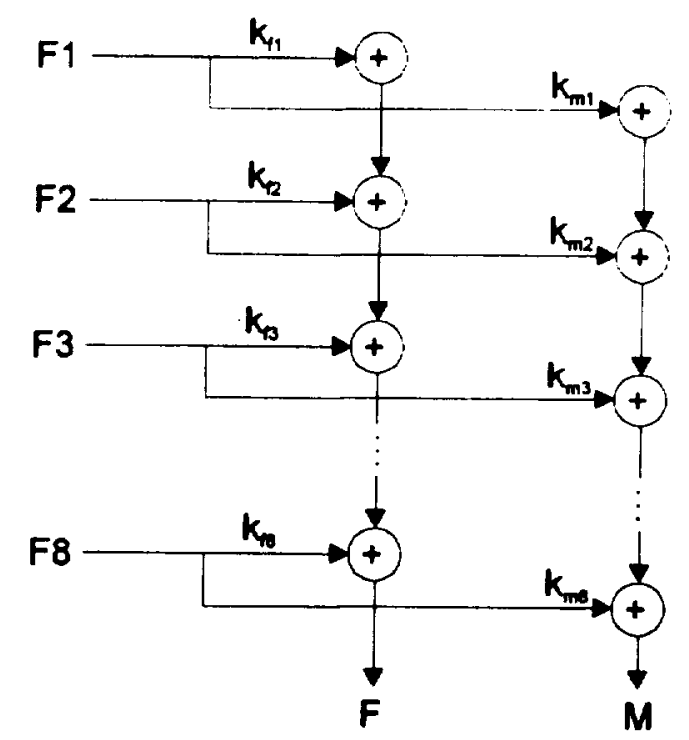

FIGURE 3. SUM, DIFFERENCE AND SCALING STAGE OF DSP BOARD

The output from the DSP board front-end is then equalized using the board's digital signal processor. A finite-impulse response (FIR) digital filter is used to perform the equalization. The FIR algorithm is shown in figure 4 . Time-delayed samples from the front-end amplifier and mixer, $x(n)$ through $x(n-N)$, are scaled by constant coefficients $a_{\text {, through }} \mathrm{a}_{\mathrm{N}}$ and summed to produce one output sample $y(n)$. This process is repeated continuously to produce a filtered, or equalized, signal. The advantages of a FIR filter include linear phase response (with proper choice of coefficients), and stability (since there is no feedback signal).

$x(n)$

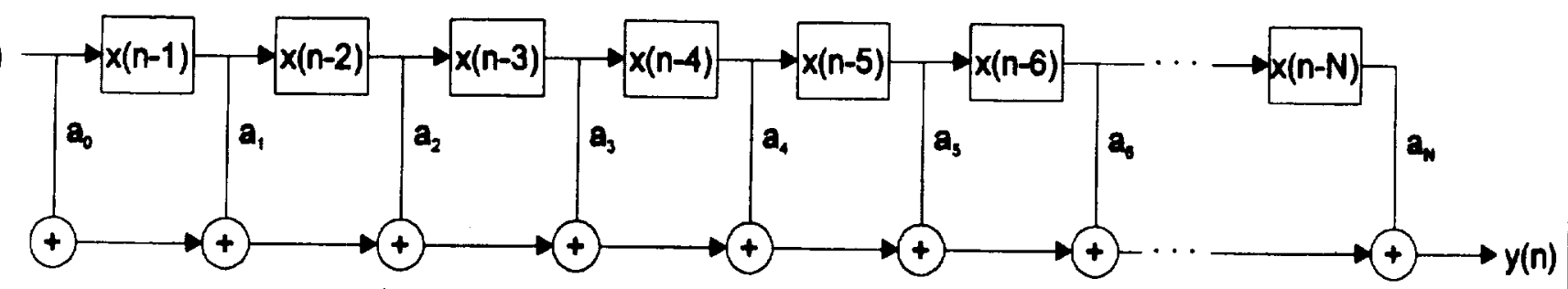

FIGURE 4. FINITE IMPULSE RESPONSE DIGITAL FILTER ALGORITHM

The equalized signal from the DSP board is then sent to the vibration control signal and is compared to the same profile used for the accelerometer signal. The only thing left to discuss is how to determine the coefficients used in the FIR equalizer.

\section{DESIGN OF THE FIR FILTER}

The steps used to design the FIR filter are summarized in table 2. These steps are discussed below using the Cryogenic InfraRed Sensor (CIRS) instrument force-limited vibration test as an example. 


\section{TABLE 2. DESIGN OF FIR FILTER}

Determine transfer function

Convert breakpoints to a real frequency spectrum

Perform IFFT

Rotate result

Truncate to a reasonable number of coefficients

Evaluate design using FFT

Test the filter

The CIRS instrument engineering test unit was tested in January and February of 1996. A photograph of the test setup is shown in figure 5. The locations of two of the six force gages are also shown in figure 5.

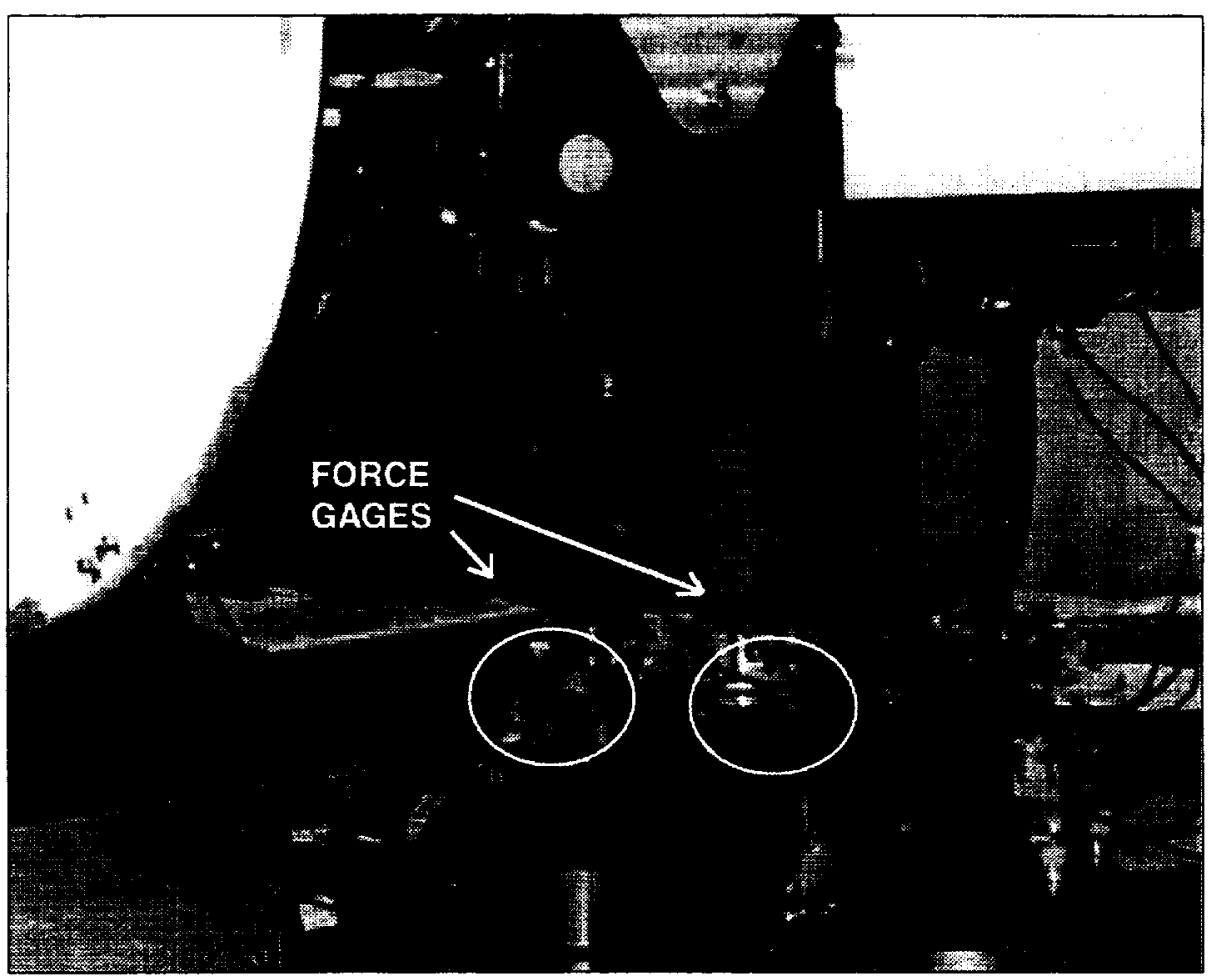

FIGURE 5. CIRS VIBRATION TEST

The CIRS instrument interfaces to the rest of the CASSINI spacecraft through a collar attached with six bolts. The bolt arrangement and the equations that determine the total forces and moments are shown in figure 6. 

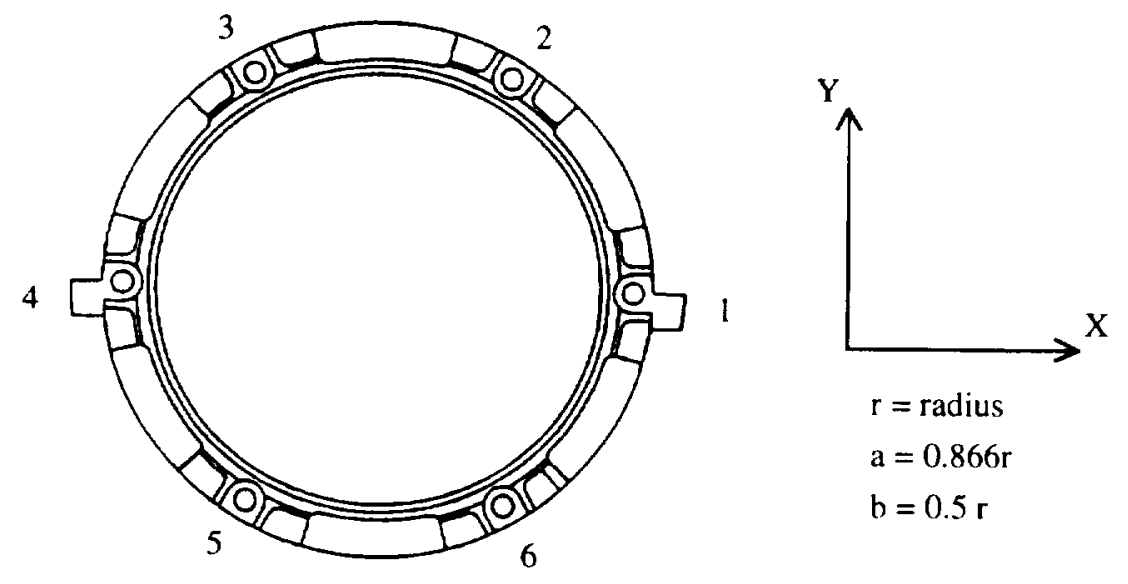

$$
\begin{aligned}
& F_{x}=F_{1 x}+F_{2 x}+F_{3 x}+F_{4 x}+F_{5 x}+F_{6 x} \\
& F_{y}=F_{1 y}+F_{2 y}+F_{3 y}+F_{4 y}+F_{5 y}+F_{6 y} \\
& F_{1}=F_{1},+F_{22}+F_{3}+F_{4}+F_{5}+F_{6,} \\
& \mathrm{M}_{\mathrm{x}}=\mathrm{aF}_{2,}-\mathrm{aF}_{s_{2}}+\mathrm{aF}_{3,}-\mathrm{aF}_{6,} \\
& \mathrm{M}_{\mathrm{y}}=-\mathrm{rF}_{1},+\mathrm{rF}_{4,}-\mathrm{bF}_{21}+\mathrm{bF}_{52}+\mathrm{bF}_{32}-\mathrm{bF}_{6 z} \\
& M_{2}=\mathrm{rF}_{1 y}-\mathrm{rF}_{4 y}-\mathrm{aF}_{2 \mathrm{x}}+\mathrm{aF}_{5 \mathrm{x}}+\mathrm{bF}_{2 \mathrm{y}}-\mathrm{bF}_{5 \mathrm{y}}-\mathrm{aF}_{3 \mathrm{x}}+\mathrm{aF}_{6 \mathrm{xx}}-\mathrm{bF}_{3 \mathrm{y}}+\mathrm{bF} \mathrm{F}_{6 \mathrm{y}}
\end{aligned}
$$

\section{FIGURE 6. CIRS COLLAR}

\section{Determine Transfer Function}

How to determine the test acceleration specification and force specifications are not the subject of this paper. Given the CIRS zdirection acceleration and force specification power spectral densities (PSD), shown in figure 7 and figure 8 respectively, the transfer function breakpoints of the filter are determined by dividing the acceleration PSD by the force PSD and taking the square root. The result is shown in figure 9. A phase of zero for the transfer function is assumed with this approach.

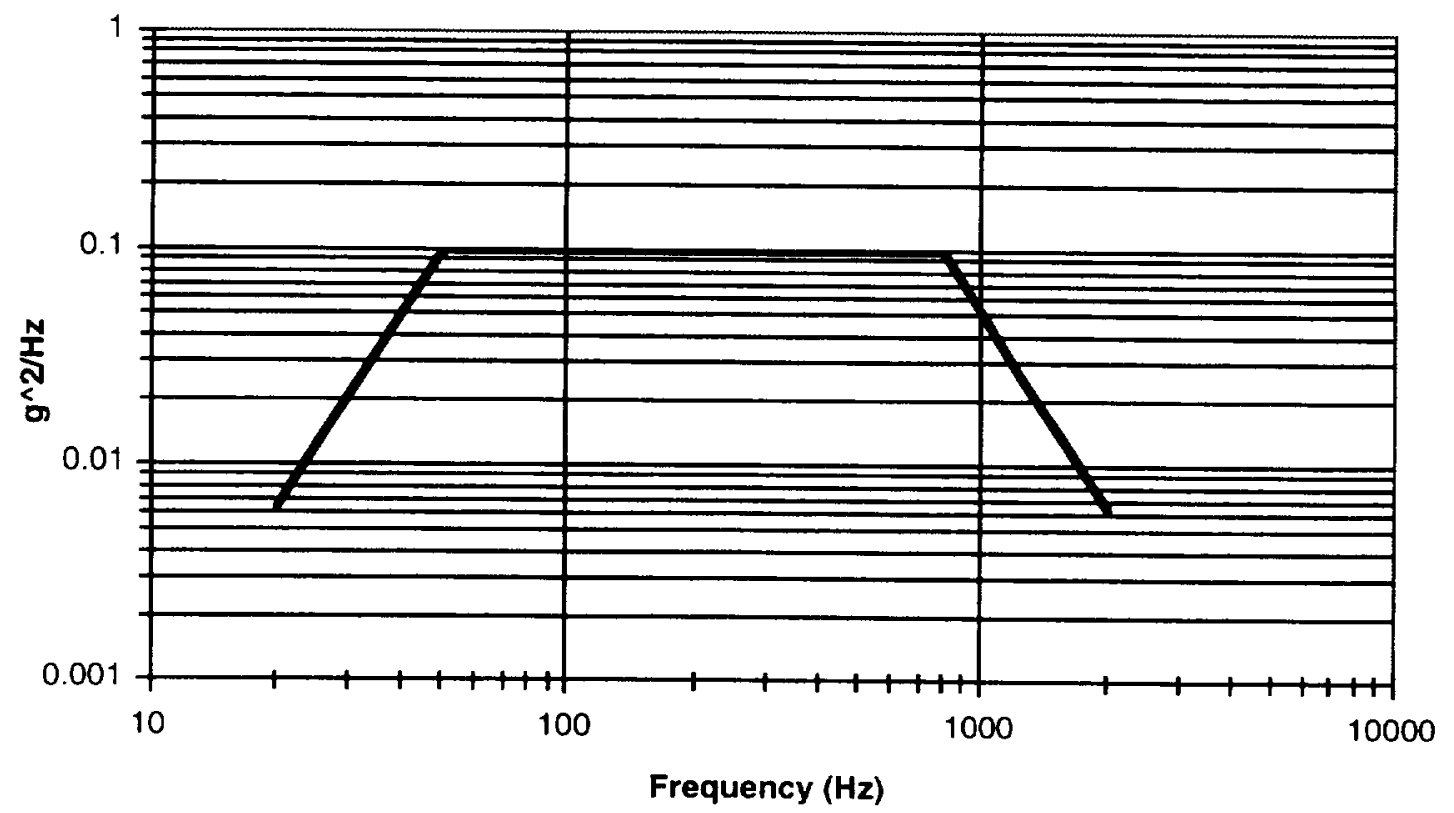

FIGURE 7. CIRS Z-DIRECTION ACCELERATION PSD SPECIFICATION 


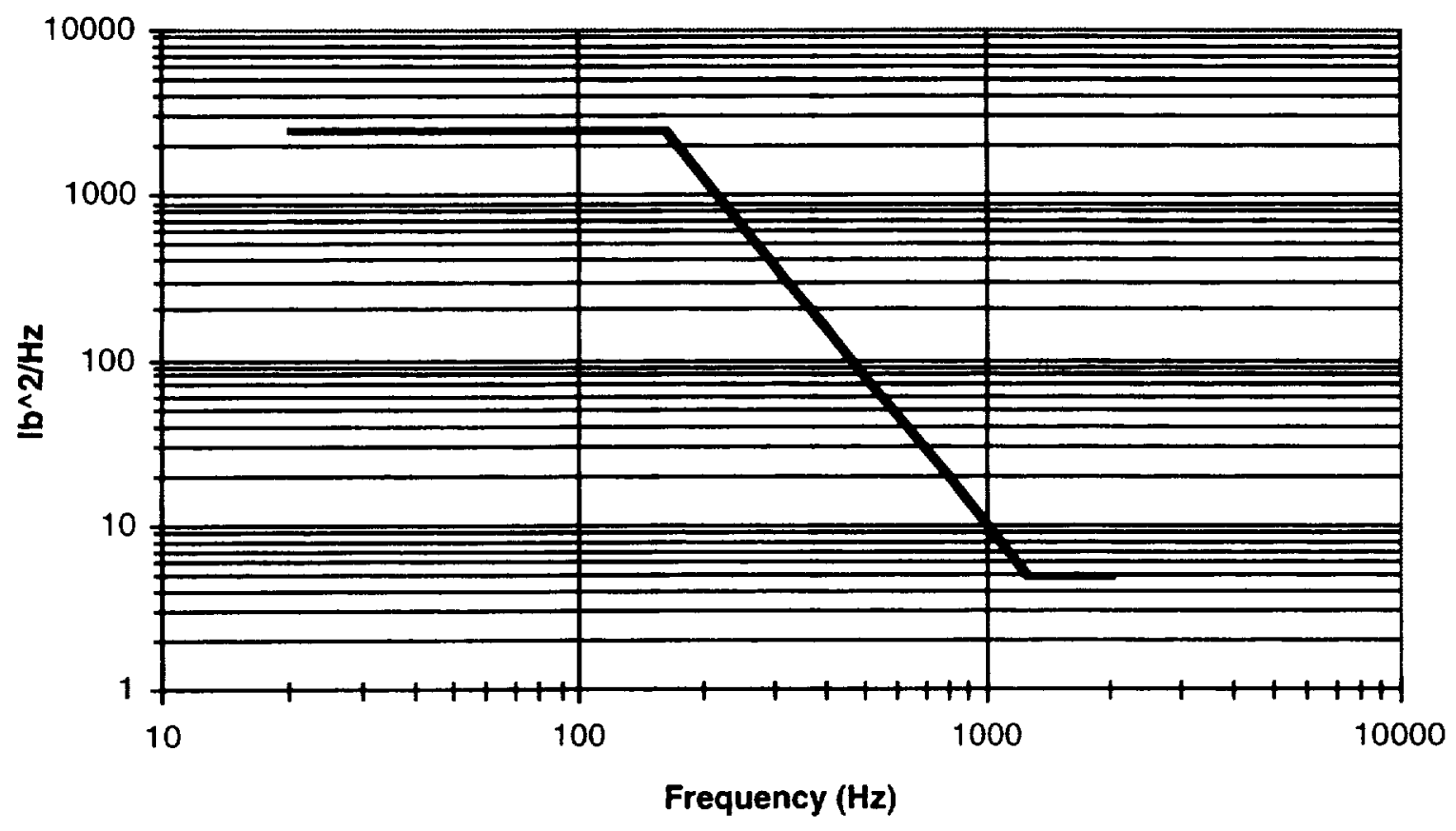

FIGURE 8. CIRS Z-DIRECTION FORCE PSD SPECIFICATION (FORCE LIMIT)

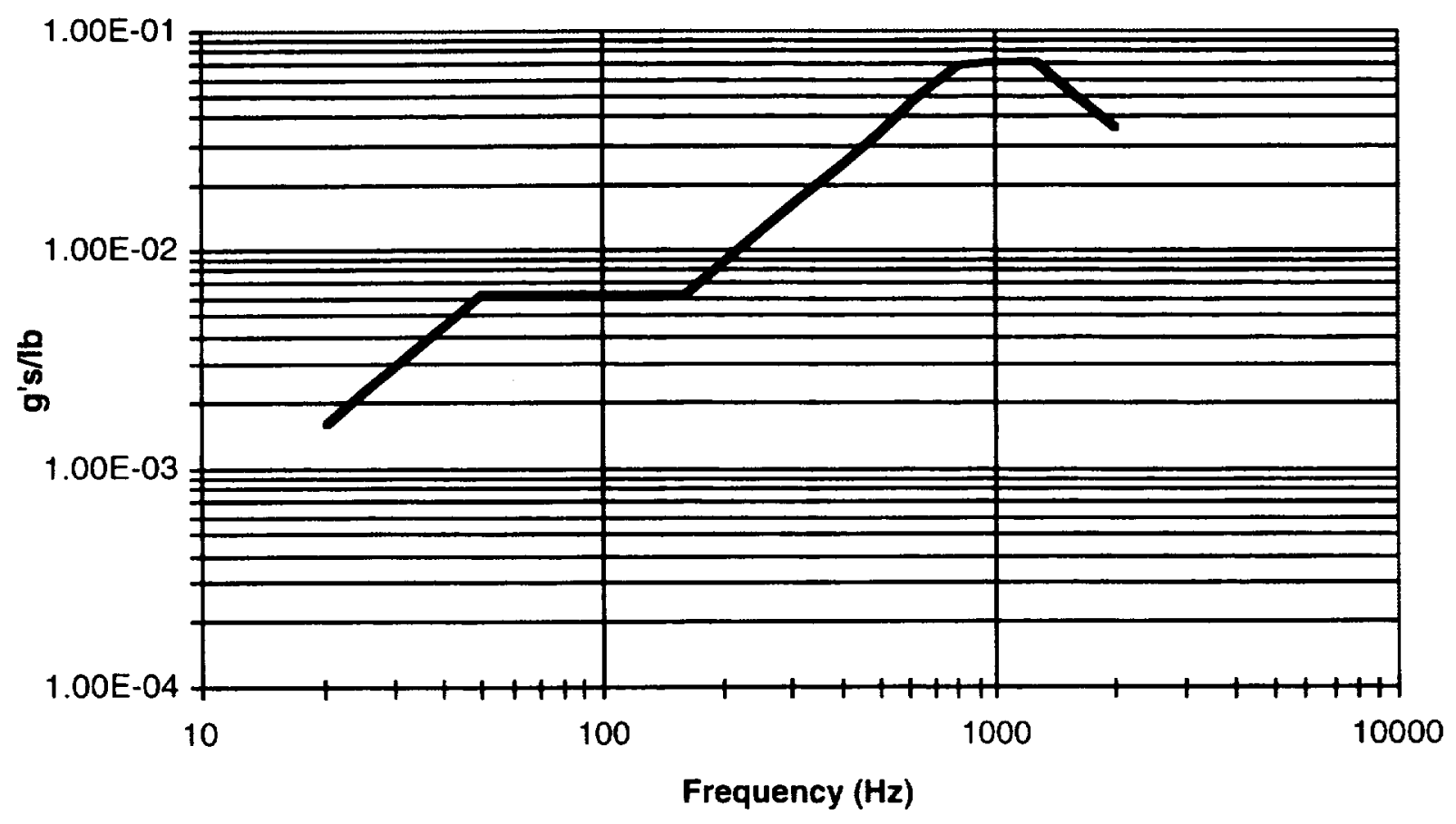

FIGURE 9. CIRS Z-DIRECTION FIR FILTER TRANSFER FUNCTION 


\section{Convert breakpoints to a real frequency spectrum}

Real (as opposed to complex) filter impulse responses produce an FFT in which the mirror image values of one half are complex conjugates of each other[4]. The right half spectrum can also be considered the negative frequency spectrum. It is also desirable to create a filter which has zero or linear phase characteristic. This is done by taking the transfer function breakpoints from the previous step, interpolating linearly spaced values, and reflecting those values into the right half of the spectrum. The resulting spectrum is shown in figure 10.

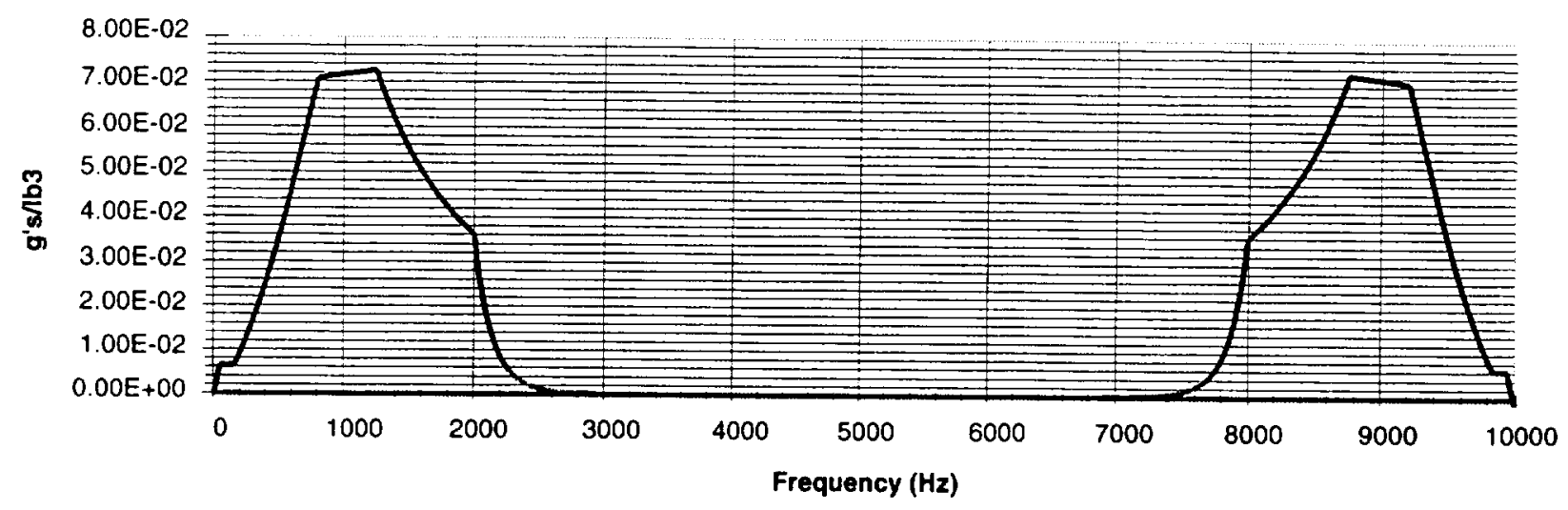

FIGURE 10. CIRS Z-DIRECTION FIR FILTER TRANSFER FUNCTION WITH NEGATIVE FREQUENCIES

This plot can be either be considered as a real spectrum with zero imaginary spectrum or a magnitude spectrum with zero phase. A zero phase spectrum is much easier to compute then a linear phase spectrum.

\section{Perform IFFT}

The impulse response of the desired filter is determined by performing an IFFT on the modified transfer function. This is shown in figure 11. One can think of the right-half of this plot as being the negative time response. This implies that the filter must have predictive behavior - that is, the response must begin before the input arrives. Because of the predictive behavior, the filter is non-realizable. Also note that most of the response occurs around zero time (or 0.04 seconds)..

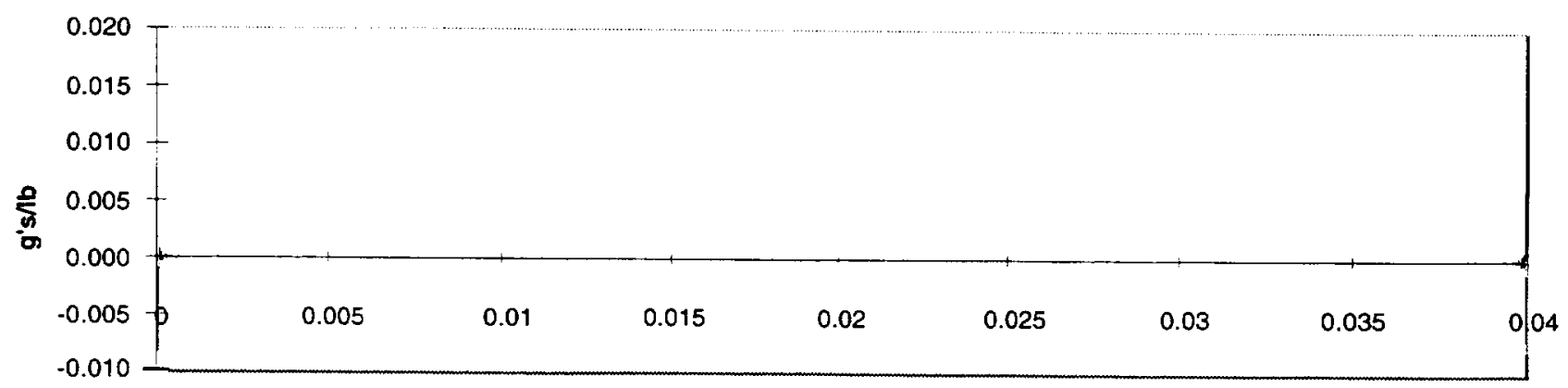

Time (seconds)

FIGURE 11. CIRS Z-DIRECTION FIR FILTER IMPULSE RESPONSE 


\section{Rotate result}

To make the filter realizable, the response is delayed (or rotated) by 20 milliseconds. The resulting filter is shown in figure 12. This filter requires $\mathbf{4 0 9 6}$ multiplications to be performed for each output sample. This would require a DSP that can perform 40 million multiplications and additions per second, which currently do not exist. The filter would also have a delay of 20 milliseconds, which is probably too much lag for a control loop.

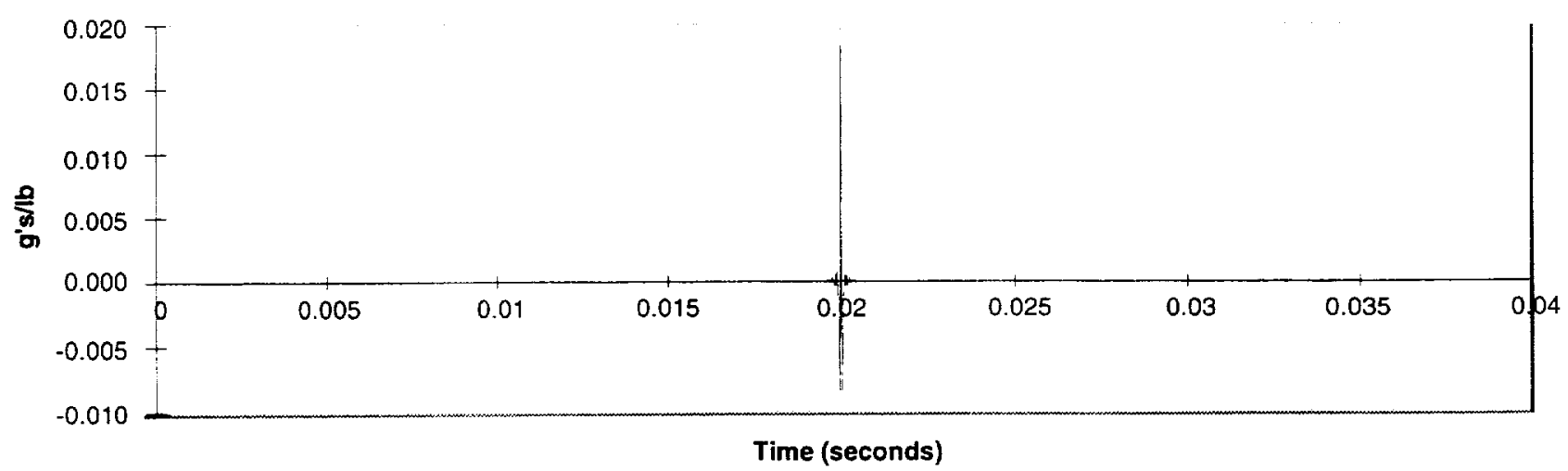

FIGURE 12. CIRS Z-DIRECTION FIR FILTER ROTATED IMPULSE RESPONSE

\section{Truncate to a reasonable number of coefficients}

Fortunately, not all of the coefficients are needed. Most of them are small compared to the ones clustered about the zero point. In this step the coefficients are truncated to a reasonable number and tested. A filter with 201 coefficients is shown on figure 13 . Since the filter has bandpass characteristics, an odd number of coefficients should be chosen [5].

\section{Coefficient Design}

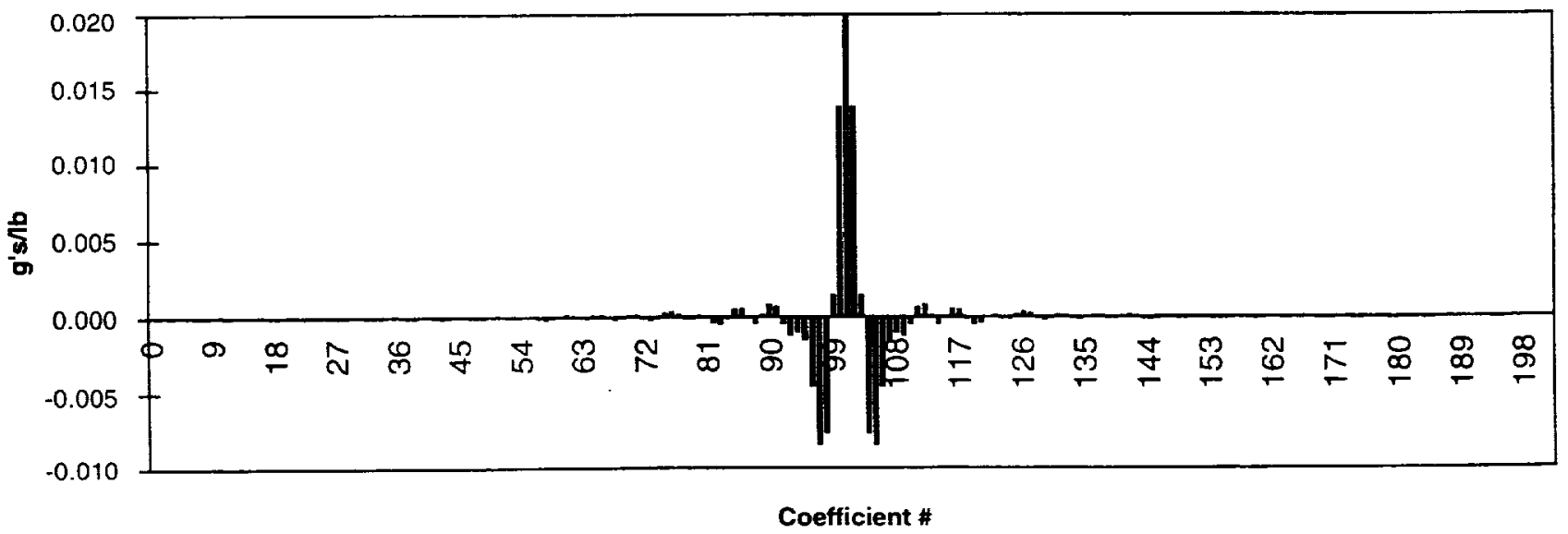

FIGURE 13. CIRS Z-DIRECTION FIR FILTER ROTATED AND TRUNCATED IMPULSE RESPONSE 


\section{Evaluate design using FFT}

Various numbers of coefficients are tried and compared to the original specification. Figure 14 shows a comparison of 201 and 511 coefficient filters. Most of the deviation is at the $50 \mathrm{~Hz}$ region of the specification curve. The 201 coefficient filter would be acceptable for this application. Since the DSP currently in use by GSFC can handle 511 coefficients before overflow, we usually use 511 coefficients.

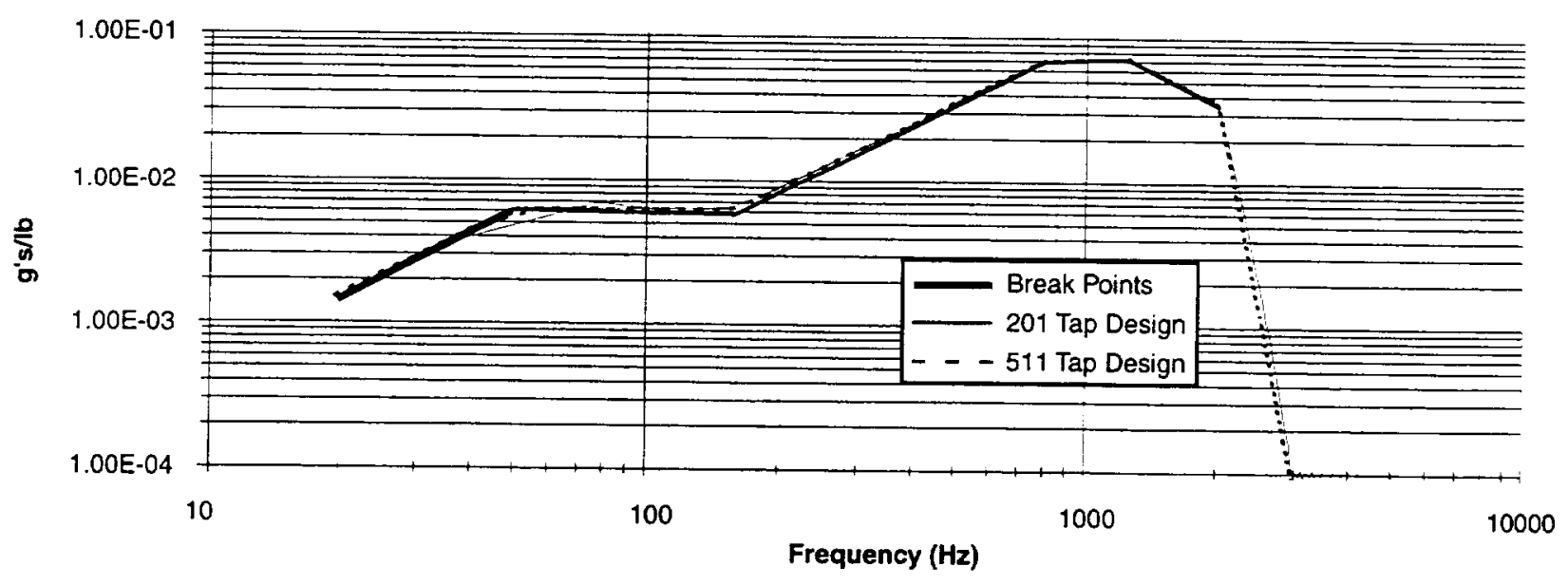

FIGURE 14. CIRS Z-DIRECTION FILTER - COMPARISON OF VARIOUS NUMBERS OF COEFFICIENTS

\section{Test the filter}

The filter characteristics are confirmed off-line by testing using a sine sweep test of the filter. At GSFC we use a Hewlett Packard 3566A FFT Dynamic Signal Analyzer to do this. A slow sweep must be used because of the significant filter delay.

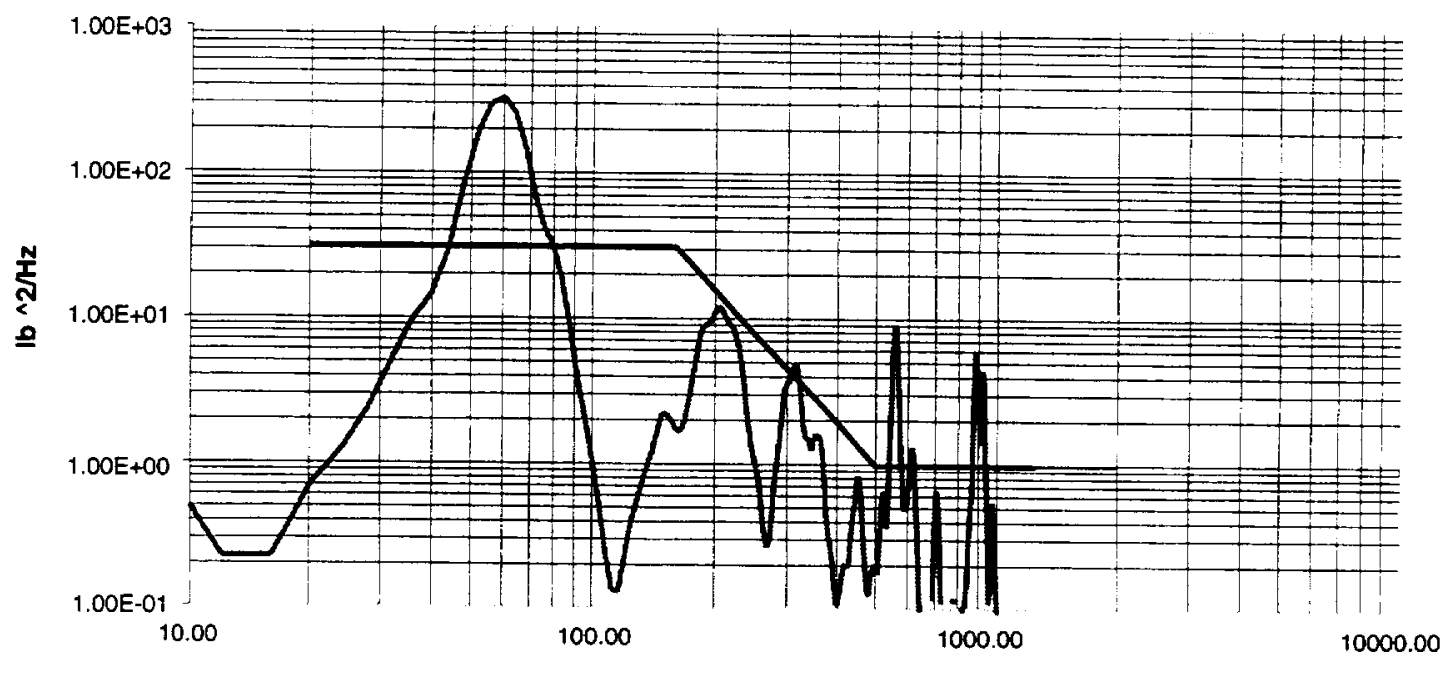

Frequency $(\mathbf{H z})$

FIGURE 15. UNEQUALIZED FORCE COMPARED TO FORCE LIMITS 
Recently GSFC has begun testing the filter during the actual random vibration test of the article. A test at low level $(-12$ or -18 $\mathrm{dB}$ ) is performed without force control. The unequalized forces or moments are compared to the appropriate force or moment limits as shown in figure 15. The equalized forces or moments are compared to the acceleration specification as shown in figure 16. Places where the force or moments limits exceed the force or moment limits are noted and compared to the exceedances on the acceleration specification. They should match exactly. In the examples shown in figure 15 and figure 16, these exceedances occur at approximately $50 \mathrm{~Hz}, 530 \mathrm{~Hz}$, and $900 \mathrm{~Hz}$.

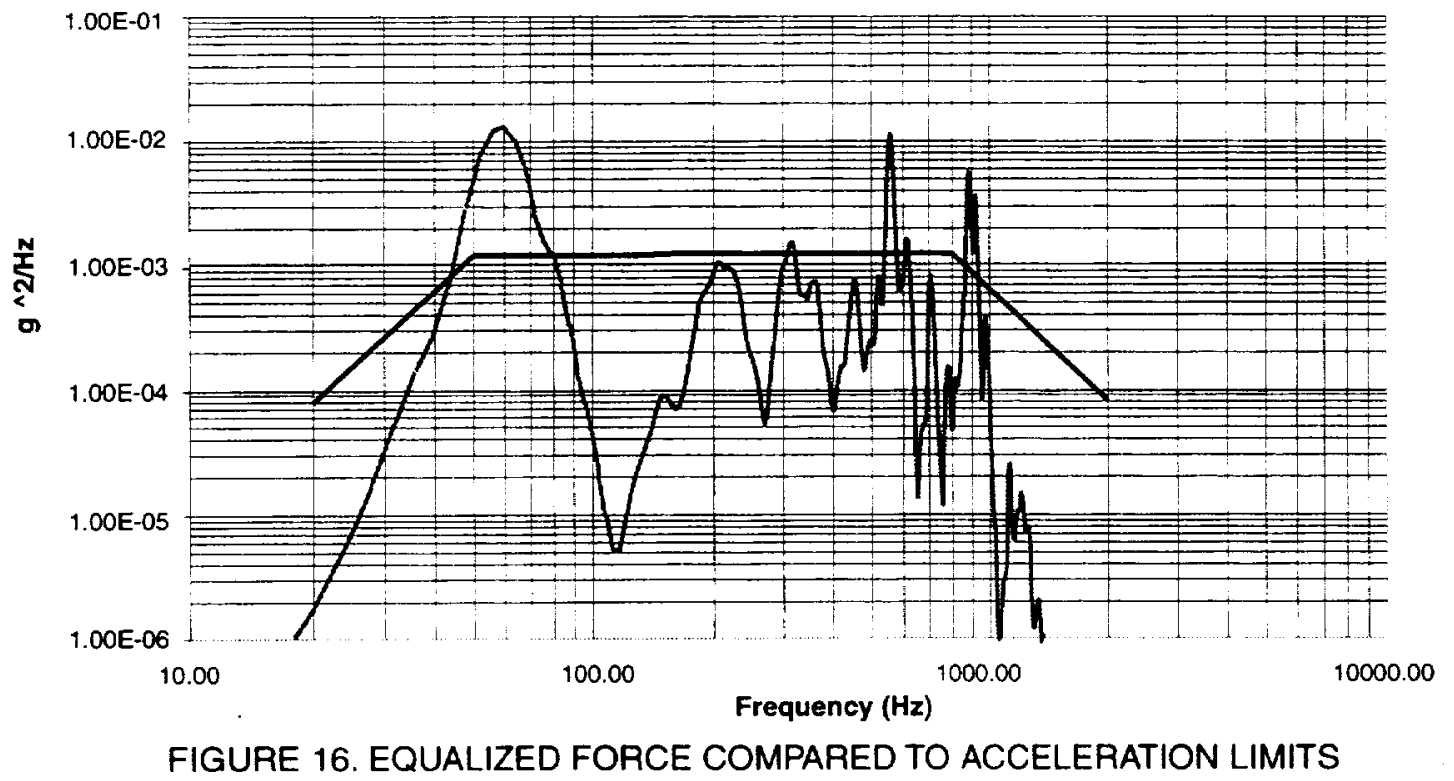

\section{Summary}

A MS-DOS program was written in the $C$ language to perform all of the steps required to design the FIR filter required to equalize the waveform. The software takes a text file containing the transfer function breakpoints as input and, with information about sampling rate and number of taps, produces another text file containing either the filter coefficients or the FFT of the coefficients for comparison purposes.

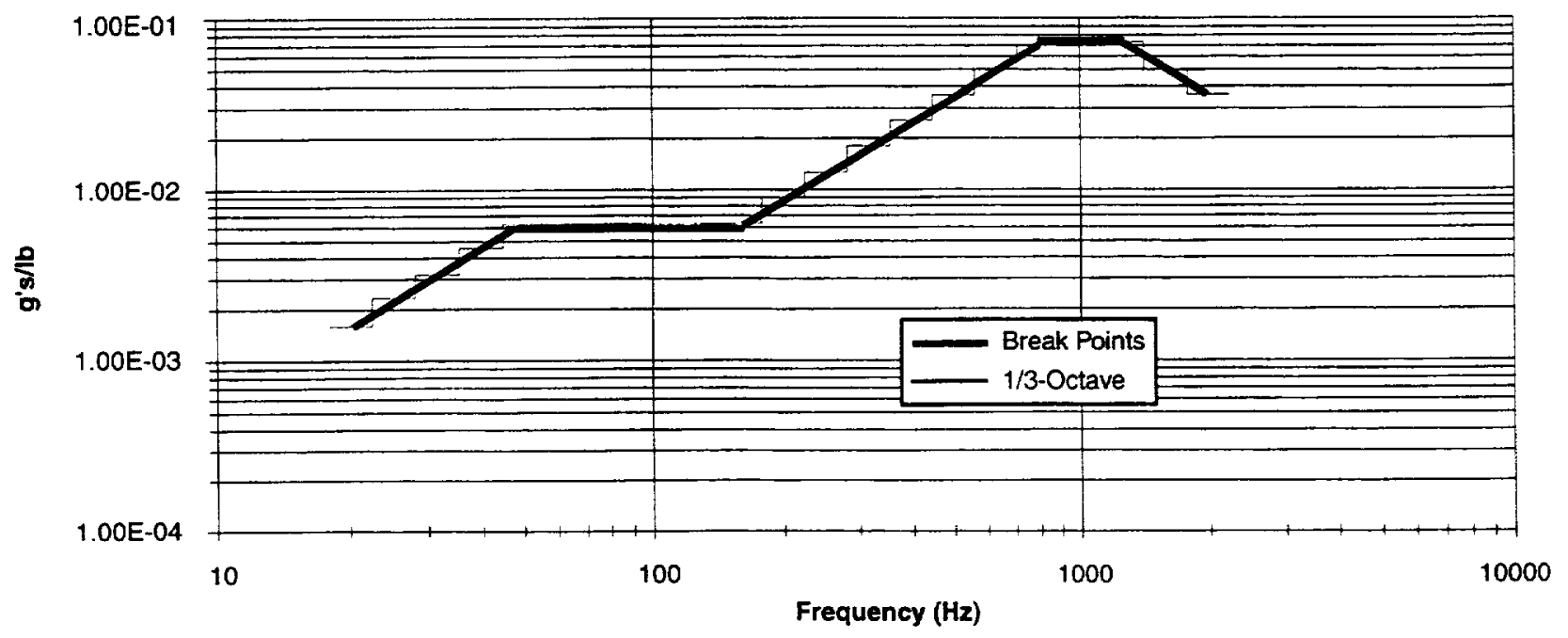

FIGURE 17. CIRS Z-DIRECTION FILTER - COMPARISON WITH 1/3-OCTAVE FILTER 


\section{CONCLUSIONS}

\section{Comparison With 1/3-Octave Filter}

Another popular means of implementing an equalizer is to use a $1 / 3$-octave filter. The resolution of such a filter is much more coarse then a DSP implementation as shown in figure 17. The figure assumes that the amplitude at each band could be set accurately which is not always the case. The minimum amplitude resolution is usually limited to $1 \mathrm{~dB}$ steps. Also, the deviation from the desired transfer function would be much worse if the slope of the curve is greater.

\section{Other Advantages}

A DSP implementation does not introduce any phase distortion. Also, the DSP board can be used for other applications such as: stepped/swept sine/cosine generator, shaped noise sources, constant force modal excitation systems, and signal analyzers. Some DSP boards have also been programmed for vibration control.

\section{Disadvantages}

The time delay of FIR filters cause problems with control strategies used for swept sine vibration which limits the equalizer filter's use to random vibration. There is also a tracking problem at low frequencies especially at higher transfer function slopes.

\section{Projects}

So far, the described system has been successfully used on the following projects:

Cassini/Cryogenic InfraRed Sensor

Mars Geophysical Surveyor/Mars Overhead Laser Altimeter II

Clark/Goddard Experimental Module

Advanced Composition Explorer

GSFC expects to be using the system described in this paper until the current controllers are replaced in the near future.

\section{REFERENCES}

1. Scharton, Terry D., "Force Limits For Vibration Tests," Spacecraft Structures and Mechanical Testing Conference Sponsored by CNES 21 June 1994.

2. Smallwood, David O., "Development of Force Envelope for An Acceleration/Force External Controlled Vibration Test," 61st Shock and Vibration Bulletin, Los Angeles, CA, October 1990.

3. Scharton, Terry D., "Vibration in Test Force Limits Derived from Frequency Shift Method," AIAA Journal of Spacecraft and Rockets, March-April, 1995.

4. Oppenhicm, Alan V. and Schafer, Ronald W., Discrete-Time Signal Processing. Prentice Hall, Inc, NJ, 1989, pp 525.

5. Loy, Nicholas J., An Engineer's Guide to FIR Digital Filters. Prentice Hall, Inc, NJ, 1988, pp 80. 


\section{.}

